\title{
Mediastinite Descendante Necrosante Aigue: Quatre Annees D'experience Dans Un Centre Hospitalier A Madagascar
}

\author{
Narindra Njarasoa Mihaja Razafimanjato \\ Tojomamy Herinjaka Ralaizafindraibe
}

USFR en Chirurgie Thoracique. Département de chirurgie à l' HU/JRA,

Tananarive, Madagascar

\section{Andriatiarainy Rija Ramarolahy \\ Tovo Andriambelo Rajaonera}

USFR en Anesthésie Réanimation. Département des sciences fondamentales

à l' HU/JRA, Tananarive, Madagascar

Jean Louis Hanitrala Rakotovao

USFR en Chirurgie Thoracique. Département de chirurgie à l' HU/JRA,

Tananarive, Madagascar

Doi: 10.19044/esj.2017.v13n33p417 URL:http://dx.doi.org/10.19044/esj.2017.v13n33p417

Abstract

Background: The acute mediastinitis also called Descending Necrotizing Mediastinitis or Cervico-mediastinitis necrotizing fasciitis is a disease which is the result of a spread of severe cervical infection down to the mediastinum. Method: A retrospective study was done at the surgical intensive care unit of Joseph Ravoahangy Andrianavalona's hospital about the management of descending necrotizing mediastinitis from $1^{\text {st }}$ January 2009 to $31^{\text {st }}$ December 2012. Result: Fourteen cases were reported during four years. The mean age of the patients was 30 years and 8 months, the sex ratio was 1.33 . The most common cause found in every cases were severe cervical infections such as fasciitis by dental origin, peritonsillar abcess, sore throat, combined with the administration of non steroid antiinflammatory, of corticoid, of inappropriate antibiotic and also the patients' health status. The suspicion of diagnosis is made clinically with chest pain associated with dyspnea, fever or septic shock and confirmed by radiologic findings. Conclusion: Nowadays, the mortality rates is high about $71,42 \%$ for our cases. Collaboration of the thoracic surgeons and anesthetists is recommended for an early trancervical drainage of the mediastinitis. In Madagascar, the fasciitis by odontogenic infection is the most common cause of mediastinitis due to the lack of dental care. 
Keywords: Abcess, Fasciitis, Mediastinum, Septic shock, Sepsis, Surgery

\section{Resume}

Introduction : La médiastinite aiguë dite également médiastinite descendante nécrosante ou cellulite cervico-médiastinale est une maladie infectieuse due à l'extension d'un foyer infectieux initialement cervical ou oro-pharyngé- Méthode : Notre étude descriptive rétrospective est réalisée chez des patients hospitalisés au sein du service de réanimation chirurgicale pour suite de prise en charge de médiastinite aiguë sur une période de quatre ans allant du $1^{\text {er }}$ janvier 2009 au 31 décembre 2012. Résultats : Quatorze cas avaient été colligés sur une période de 4 ans. La moyenne d'âge des malades était de 30 ans et 8 mois avec prédominance masculine, (sex ratio $=$ 1,33). Les principaux facteurs de risque retrouvés étaient la présence d'une infection oro-pharyngée sévère surtout de cellulite d'origine dentaire, la notion de prise d'anti-inflammatoire non stéroïdiens, de corticoïdes, d'antibiotiques et le terrain du patient. Le diagnostic est clinique faite de douleur thoracique associée à une dyspnée type orthopnée, fièvre voire à un état de choc septique et confirmé par les examens paracliniques et parfois un diagnostic per-opératoire. Dans cette étude, le traitement était pluridisciplinaire fait de réanimation et de traitement chirurgical par une cervicotomie large ou nécessitant rarement une stérnotomie. Conclusion : L'évolution actuelle est surtout marquée par un décès dans $71,42 \%$. A Madagascar, la négligence de l'état bucco-dentaire source de cellulite d'origine dentaire joue un rôle très prépondérant dans l'apparition de la médiastinite descendante nécrosante aiguë.

Mots clés : Abcès, Cellulite, Chirurgie, Choc Septique, Médiastin, Sepsis.

\section{Introduction}

La médiastinite descendante nécrosante aiguë (MDNA) est une pathologie due à une extension de l'infection cervico-faciale ou oropharyngée sévère au niveau du médiastin (Doddoli, 2009). Elle constitue une urgence de prise en charge diagnostique et thérapeutique. C'est une pathologie grave qui menace le pronostic vital à court terme avec un taux de mortalité très élevé (20 à 40\%) suite au retard de diagnostic (Doddoli, 2009), contre $50 \%$ en 1938 où le principal problème était la non découverte de l'antibiothérapie (Biland, 1999). L'intérêt de cette étude porte sur la constatation d'une augmentation de l'incidence de médiastinite descendante nécrosante aiguë avec un taux de mortalité élevé dans le service de réanimation chirurgicale et du service de Chirurgie thoracique du CHUAHUJRA. 
Nos objectifs sont d'analyser les aspects chirurgicaux des médiastinites descendantes nécrosantes aigues afin d'évaluer son traitement en milieu précaire et améliorer la morbi-mortalité liée à cette pathologie.

\section{Patients et methode}

Pour évaluer le traitement des médiastinites aigues, nous avons mené une étude descriptive transversale des cas de médiastinite descendante nécrosante aiguë sur une période de quatre ans allant du 01 Janvier 2009 au 31 Décembre 2012, réalisée dans le service de Réanimation Chirurgicale de l'Hôpital Universitaire Joseph Ravoahangy Andrianavalona du Centre Hospitalier Universitaire d'Antananarivo (HU/JRA).

Cette étude est exhaustive de tous les patients ayant été pris en charge pour médiastinite aigue descendante d'origine ORL et dentaire et possédant un dossier complet pour orienter le diagnostic en préopératoire. Ainsi nous avons exclu de ce travail les patients présentant une médiastinite post-opératoire et les patients dont les dossiers sont incomplets.

\section{Resultats}

Durant la période d'étude, nous avons colligé 14 cas de MDNA avec une fréquence annuelle représentée par la figure ci-dessus (Figure 1).

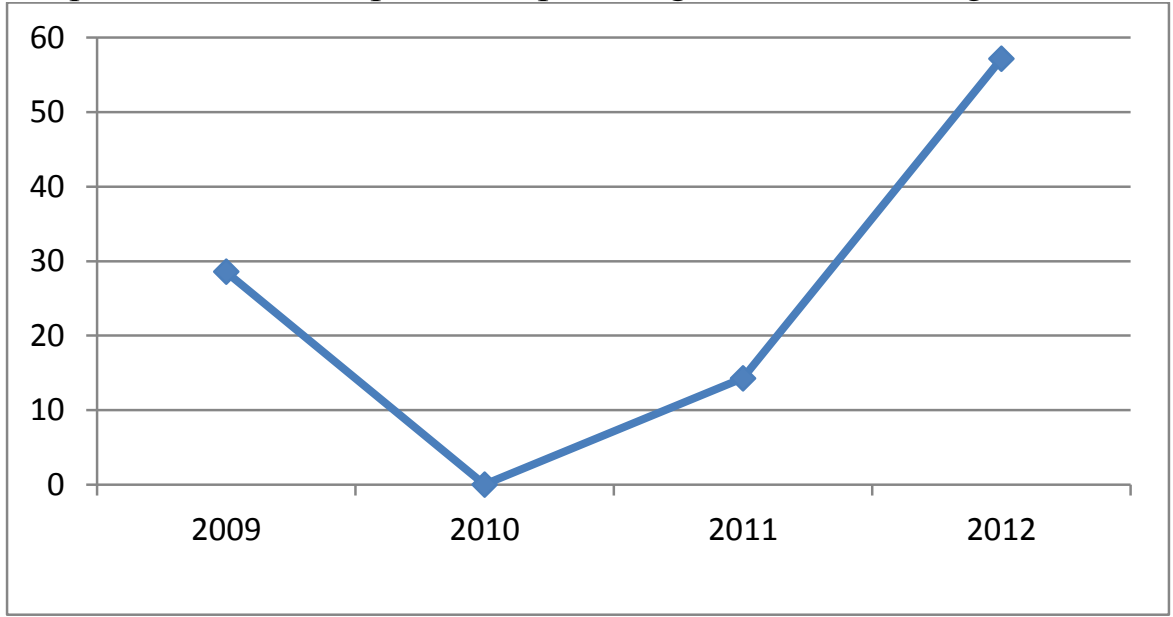

Figure 1: Courbe de la fréquence annuelle de médiastinite aiguë opérée dans notre centre.

L'âge médian était de 30 ans et 8 mois avec un extrême entre 3 ans et 62 ans. Le sex-ratio était de 1,33 (6 femmes et 8 hommes). Le délai diagnostic variait de 3 jours à 60 jours avec un délai moyen de 18 jours. Dans notre étude, nous avons relevé dans les antécédents deux patients diabétiques (14,28\% des cas), 8 cas de dénutrition sévère ( $57,14 \%$ des cas), 4 alcooliques $(28,57 \%$ des cas). La prise d'anti-inflammatoire non stéroïdien était notée dans 6 dossiers de patient (42,85\% des cas), 2 malades ayant pris 
des antibiotiques $(14,28 \%$ des cas) et 2 cas de corticothérapie $(14,28 \%$ des cas) avant l'hospitalisation.

Le tableau clinique était polymorphe, les symptômes motivant la consultation et l'hospitalisation des patients sont récapitulés avec leurs fréquences respectives dans le tableau I.

Tableau I: Récapitulatif de la répartition des patients selon la symptomatologie.

Signes fonctionnels observés à l'admission

Dyspnée

Dysphagie

Douleur thoracique

Dysphonie

Signes digestifs
Effectif $(\mathrm{N})$

10

6

2

2

4
Taux $(\%)$

71,42

42,85

14,28

14,28

28,56

L'altération de l'état général était notée chez 10 patients $(71,42 \%)$, et un assez bon état général dans le reste des cas (28,57\%). La fièvre était retrouvée chez 12 patients $(85,71 \%), 02$ patients étaient apyrétiques (soit $14.28 \%$, et un sepsis sévère chez 10 patients $(71,42 \%$ des cas $)$. La tachycardie a été notée chez 10 patients $(71,42 \%)$ et était normal chez 4 patients $(28,57 \%)$.

L'examen pleuro-pulmonaire a objectivé un syndrome d'épanchement liquidien chez 8 malades $(57,14 \%)$ et gazeux pour 6 malades (42,85\%), dyspnée chez 10 patients $(71,42 \%)$, des signes de lutte chez 2 malades $(14,28 \%)$. Un œdème inflammatoire au niveau de la région cervicothoracique a été observé chez 12 patients dans notre série (soit 85,71\% des cas). Une fistulisation cutanée était notée dans 2 cas (soit 14,28\%).

L'examen bactériologique avec mise en culture du prélèvement effectué au niveau du foyer infectieux a été réalisé chez 2 malades $(14.28 \%$ des cas) et a retrouvé comme germe un enterobacter cloacae et du citrobacter freundii multi résistant.

Dans notre série de cas, la radiographie thoracique de face a objectivé les signes radiologiques avec leurs incidences résumés dans le tableau II.

Tableau II : Tableau résumant les signes radiologiques observés à l'entrée des patients

$\begin{array}{lll}\text { Signes radiologiques } & \text { Effectif }(\mathrm{N}) & \text { Taux }(\%)\end{array}$

Epanchement pleural liquidien unilatéral

Pneumothorax
8

4
66,66

33,33 
Hydropneumothorax

Emphysème pariétal ou médiastinal

Elargissement du médiastin supérieur à limites floues

«V » de Naclério
16,66

50

16,66

16,66

Le scanner thoracique a été réalisé chez 4 patients $(28,57 \%$ des cas $)$. La TDM a mis en évidence des épanchements siégeant au niveau de la plèvre pour les 4 cas avec 1 cas de grande abondance, un autre scissural, et un épanchement péricardique dans 2 autre cas (50\%). Une collection liquidienne sans niveau hydro-aérique au niveau du médiastin a été objectivée dans 2 cas $(50 \%)$, ainsi qu'un de pneumomédiastin; la densification de la graisse médiastinale a été retrouvée dans 3 cas.

L'origine de la MDNA dans notre série est récapitulée dans le tableau III selon le siège et la nature de lésion retrouvée.

Tableau III : Tableau résumant les étiologies des MDNA retrouvées dans notre série.

\begin{tabular}{cccc}
\hline Siège & Lésions & Effectif $(\mathrm{N})$ & Taux (\%) \\
\hline $\begin{array}{c}\text { Stomatologie } \\
\text { Oropharyngé }\end{array}$ & $\begin{array}{c}\text { Cellulite dentaire } \\
\text { Phlégmon péri- } \\
\text { amygdalien } \\
\text { Angine ulcéro- } \\
\text { nécrosante } \\
\text { Eerforation } \\
\text { Esophage }\end{array}$ & 4 & 50 \\
& $\begin{array}{c}\text { esophagienne } \\
\text { (dentier) }\end{array}$ & 1 & 14,28 \\
\hline
\end{tabular}

Concernant la prise en charge, tous les patients ont bénéficié d'une prise en charge selon un protocole validé dans notre centre comportant des mesures de réanimation et une antibiothérapie probabiliste et adaptée selon l'antibiogramme. Dans notre série, l'antibiothérapie était adaptée dans les deux cas (14,28\% des cas) ou on a trouvé des BMR (Bacille multi-résistant) dans les prélèvements de pus. Dans le reste des cas (soit 85, 71\%), elles étaient adaptées en fonction de l'évolution clinique. Les molécules utilisées étaient les dérivés de Pénicilline dans 14 cas (100\%), associés à un imidazolé dans 12 cas $(85,71 \%)$, à un aminoside dans 6 cas $(42.85 \%)$ et à une quinolone dans les 4 cas.

Le traitement chirurgical pour la nécrosectomie et le drainage a été réalisé chez 12 malades $(85,71 \%$ des cas) et non indiqué chez deux malades (14,28\% des cas) en raison d'une contre-indication anesthésique. Il s'agissait d'une cervicotomie large seule dans 10 cas (soit 83,33\%) et associée à une sternotomie dans 2 cas de patient (soit 16,66 \%) (Photo 1). L'alimentation 
des patients était assurée par voie entérale à l'aide d'une sonde nasogastrique mise en place de façon systématique en peropératoire.

La durée d'hospitalisation varie de 2 à 30 jours avec une moyenne de 9 jours et dont 1 à 19 jours en service de réanimation soit une moyenne de 5 jours.

Les deux malades n'ayant pas bénéficié du traitement chirurgical sont décédés $(14,28 \%)$. Au total, nous avons observé 4 cas d'évolution favorable ( $28,45 \%$ des cas), 8 malades sur 12 post-opérés sont décédés suite à une défaillance multiviscérale et un état de dénutrition sévère $(66,66 \%$ des cas). Le décès étant survenu entre moins de 24 heures à 7 jours post-opératoires avec une moyenne de 2,43 jours.

\section{Discussion}

Selon la littérature, il s'agit d'une pathologie rare (Alsoub, 1995). Néanmoins, une étude scannographique réalisée sur une période de 8 ans avait rapporté une série de 130 patients ayant présenté une fasciite nécrosante cervicale associée à une médiastinite dans $100 \%$ des cas (Petitpas, 2012).

Notre étude rétrospective de quatre ans portant sur les cas de MDNA démontre la rareté de cette pathologie, 14 cas en 4 ans, par rapport aux hémorragies digestives qui constituent les premiers motifs d'hospitalisation dans notre service de réanimation chirurgicale suivies des traumatismes crânio-encéphaliques. Nous avons déduit que l'incidence en hausse de cette pathologie à partir de 2010 s'explique par le contexte politique et socioéconomique de Madagascar depuis 2009 répercutant sur l'état de santé de la population en l'occurrence par négligence des infections oropharyngées et des soins bucco-dentaires. Ceci se justifie par le manque de moyens de la population entrainant la baisse du taux de consultation spécialisée, une négligence à l'origine du taux grimpant de MDNA constaté en 2012 dans cette étude.

Dans notre série nous avons noté des âges extrêmes de 3 ans et 62 ans, avec une moyenne de 30 ans et 8 mois. Par rapport à la littérature (Chinh, 2003), cet âge médian peu élevé s'explique par le fait que nos patients sont surtout constitués par une proportion relativement élevée de jeunes adultes, reflétant la moyenne d'âge de la population à Madagascar. La prédominance masculine notée dans notre étude est maintes fois rapportée dans la littérature et s'explique par l'incidence deux fois plus élevée chez les hommes de l'alcoolo-tabagisme.

Dans une étude effectuée par Petitpas et al, il note un délai moyen d'admission au service des urgences chirurgicales de 4 jours étant donné que les patients ont subi un traitement soit en ambulatoire par des médecins généralistes soit dans un autre service (Petitpas, 2012). Notre étude a 
retrouvé un délai moyen de diagnostic de 15 jours chez $71,42 \%$ des cas. L'hospitalisation à Madagascar s'avère être de dernier recours, il y a une nette préférence pour le traitement en ambulatoire voire même pour la médecine traditionnelle. L'ignorance et l'éloignement géographique de certaines régions par rapport au centre de santé sont des facteurs favorisant à ce retard. De plus, notre pays ne dispose pas de système de sécurité sociale approprié pour financier la prise en charge médicalisée des patients à l'hôpital.

Dans notre étude, les 2 cas de MDNA secondaires à une infection oropharyngée $(28,57 \%$ des cas) font suite à une automédication par des antiinflammatoires non stéroïdien et d'antibiotique et un traitement médical en ambulatoire à base de corticoïde qui s'est avéré non efficace. L'origine de la négligence d'une pathologie à Madagascar est multifactorielle. Nous avons surtout noté les facteurs financiers et psycho-éducatifs outres les autres facteurs de risques classiques retrouvés dans la littérature comme l'alcoolisme, le tabagisme, le diabète, la malnutrition et l'état d'immunodépression (Razafimanjato, 2012 ; La Rosa J, 2008).

Sur le plan clinique, nos patients sont vus le plus souvent à un stade assez tardif et toutes les différentes symptomatologies décrites dans la littérature ont été retrouvés. Cette situation tardive a été objectivée dans certaines littératures où les patients sont vus par les chirurgiens thoraciques de façon tardive après installation de défaillance multiviscérale (Foroulis, 2011).

Sur le plan biologique, l'examen bactériologique du pus n'a pas pu être réalisé pour la majorité de nos patients, ce qui ne permettait pas l'antibiothérapie adaptée en post-opératoire. Ceci s'explique outre le problème financier auquel font face la plupart de nos patients (soit 57,14 \%) par le fait que nous ne disposons pas dans notre établissement un service de bactériologie de garde pour la transmission rapide du prélèvement au bloc la nuit et les weekends. Pourtant, cette étape diagnostique est directement correlée au pronostic de cette pathologie, permettant une adaptation de l'antibiothérapie après identification des germes et antibiogramme après culture (Petitpas, 2012 ; Serghini, 1995).

Dans notre étude, la radiographie cervico-thoracique est l'examen morphologique le plus effectué, et reste de première intention dans note pratique. C'est le seul examen accessible par le pouvoir d'achat des patients dans notre établissement et nos diagnostics reposaient sur la radiographie du thorax dans $85,71 \%$ des cas. Les littératures sont controversées, certains auteurs rapportent que cet examen n'est pas très utile dans l'évaluation ou le diagnostic de la médiastinite car ne montre que des signes tardifs (Mathieu, 1995). 
D'autres auteurs rapportent qu'il peut objectiver un élargissement du médiastin supérieur dans $40 \%$ des cas, un épanchement pleural uni ou bilatéral aspécifique dans $40 \%$ des cas, un emphysème sous cutané et/ou médiastinal dans $30 \%$ des cas, un niveau hydro-aérique médiastinal et épanchement péricardique dans 10\% des cas (Doddoli, 2009).

Le scanner thoracique permet le diagnostic précoce de la médiastinite et également sa classification orientant alors le choix de l'abord chirurgical approprié ainsi que la surveillance en post-opératoire (Giudicelli, 2003). Dans notre travail, le scanner n'a pu être réalisé que chez 2 patients à cause du coût encore exorbitant de cet examen dans les pays en développement comme Madagascar. De plus cet examen est encore considéré comme un examen superflu dans les préjugés populaires à Madagascar.

Concernant l'étiologie de cette pathologie, la littérature incrimine surtout l'infection dentaire et oropharyngée comme étant les principales causes à s'étendre au niveau du médiastin (Hirai, 2004). Ceci est vérifié dans notre étude tout en sachant que $71,42 \%$ de nos patients étaient référés par le service d'ORL, Stomatologie et Chirurgie Maxillo-Faciale. Dans d'autre étude faite au Vietnam, l'étiologie était surtout dominée par la perforation œsophagienne par ingestion de corps étranger alimentaire (Chinh, 2003). Dans les pays développés, les infections médiastinales (médiastinite aiguë ou abcès médiastinal) se voient surtout après une chirurgie cardiaque (Durrleman, 2006).

Sur le plan thérapeutique, une étude rapporte un délai de diagnostic très significatif impactant sur le pronostic vital des patients (Doddoli, 2009). Selon certains auteurs, le délai entre le diagnostic et le traitement devrait être de moins de $24 \mathrm{~h}$ pour obtenir un bon résultat (Foroulis, 2011). Notre étude rapporte un diagnostic tardif de 57 heures lié au retard de réalisation des explorations étant donné les problèmes financiers des patients. Dans notre pratique, l'indication opératoire par rapport à la prise en charge des patients est fondée sur des arguments anamnestiques et cliniques. Les voies d'abord chirurgical pour aborder le médiastin dans cette pathologie reste un sujet de controverse. Une méta-analyse publiée par Corsten et al. en 1997, rapporte que le médiastin ne peut être drainé par une chirurgie limitée par la voie xyphoïdienne ou par une médiastinostomie (chirurgie mini-invasive), la thoracotomie est la meilleure pour l'exploration allant de l'œsophage à l'espace pré-vertébral qui devrait être effectué minutieusement (Foroulis, 2011). D'autres auteurs affirment que la chirurgie doit toujours commencer par un drainage cervical (Doddoli, 2009).

Dans notre pratique, le choix de cette voie d'abord repose sur des critères clinico-radiologiques et évolutifs de la maladie. La cervicomédiastinostomie large était pratiquée dans tous les cas. Cette dernière nous a permis de pratiquer une nécrosectomie large de la région cervicale et de 
drainer la cavité médiastinale. Un double abord cervical et xiphoïdien serait choisi dans le cas de MDNA dépassant la carène sur la radiographie du thorax de profil. La deuxième voie d'abord nous permet de drainer la cavité péricardique en cas de péricardite associée. Un drainage thoracique percutané complétait les gestes chirurgicaux en présence de pleurésie purulente. Une gastrostomie d'alimentation n'était pas systématique du fait que nous n'avons pas relevé de perforation œsophagienne chez nos patients.

Notre taux de létalité était de $71,42 \%$, soit 10 patients morts sur une série de 14 hospitalisés et pris en charge pour médiastinite. Un taux de mortalité très élevé par rapport à la littérature. Pour les formes localisées, Gobien et al. ont publié cinq succès sur six malades traités et Nakamori et al ont eu 100\% de succès chez 20 malades traités (Biland, 1999). Dans la majorité des cas, le retard de prise en charge et le traitement chirurgical non optimal sont les facteurs principaux de surmortalité dans la médiastinite aiguë. Dans notre contexte, les patients arrivent dans un service spécialisé seulement à un délai moyen de 18 jours témoigné par les deux cas de fistulisation cutanée (Photo2). Deux patients n'ont pas pu bénéficier de la chirurgie en raison de leur mauvais état général lié à une défaillance multiviscérale à l'admission.

\section{Conclusion}

La fréquence de la médiastinite descendante nécrosante aiguë s'est élevée ces trois dernières années. La cellulite d'origine dentaire en est la cause la plus incriminée vue la négligence sur l'état bucco-dentaire très marquée dans notre population.

Dans notre pratique, la cervicotomie reste un moyen à la fois diagnostique pour une classification de la médiastinite et thérapeutique. Le taux de mortalité de la MDNA retrouvé dans cette étude était d'environ $71,42 \%$ des cas étudiés, la diminution de cette surmortalité nécessite une bonne coopération entre les autorités administratives, les personnels soignants et la population pour la prévention de sa survenue et l'amélioration de la prise en charge de la pathologie ainsi que du pronostic. 


\section{Illustrations :}

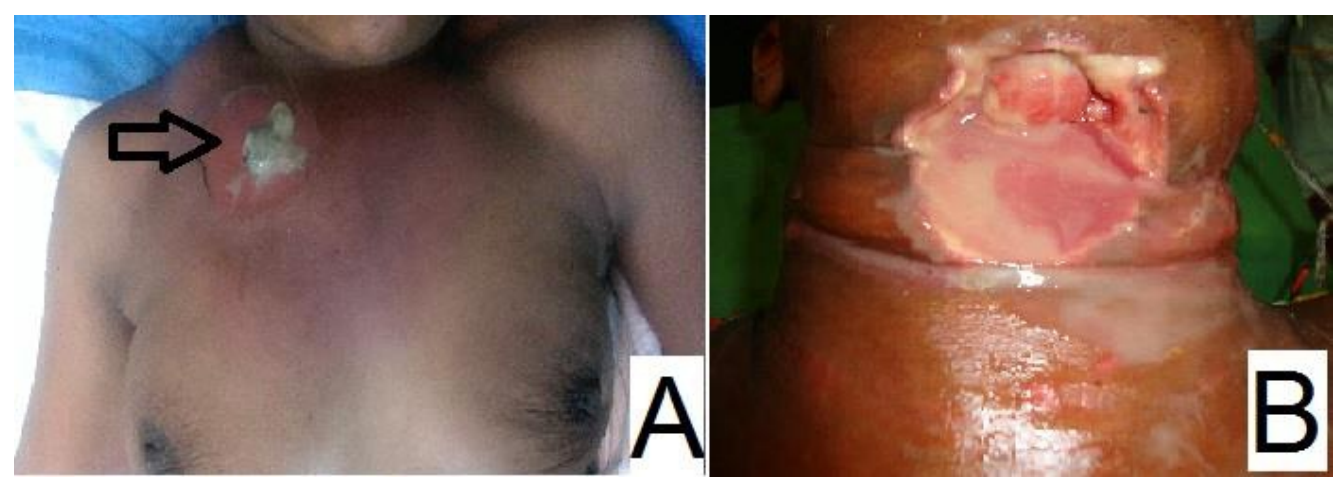

Photo 1:

A : Fistulisation cutanée au niveau de la paroi antérieure du thorax d'une cervicomédiastinite aiguë (Flèche noire)

B : Patient présentant une cervico-médiastinite d'origine dentaire fistulisée au niveau de la région cervicale antérieure opéré par une cervicotomie large.

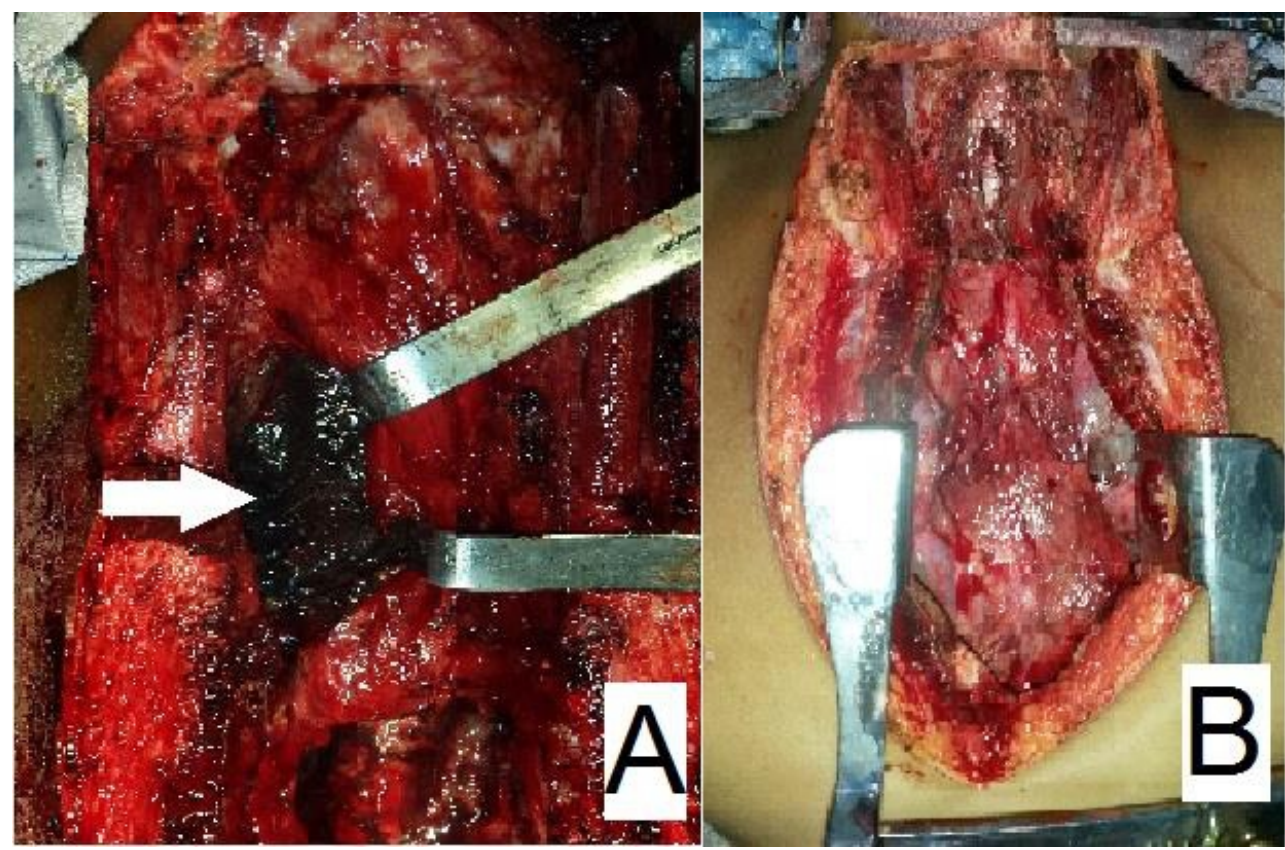

Photo 2 :

A : Photo peropératoire d'un patient montrant une médiastinite descendante nécrosante aiguë (MDNA) (Flèche blanche)

$\mathrm{B}$ : Photo peropératoire du même patient après nécrosectomie de la région cervicomédiastinale antérieure 


\section{References:}

1. Doddoli, C., Trousse, D., Avaro, J. P., Djourno, X. B., Jaussaud, N., Giudicelli, R., ... \& Thomas, P. (2009). Traitement des médiastinites descendantes nécrosantes aiguës. EMC.

2. Biland, G., Delcambre, F., Jougon, J., \& Velly, J. F. (1999). Médiastinites descendantes opérées par cervico-sternotomie: expérience chirurgicale de 13 ans. Cardiovasc Surg, 47, 14-9.

3. Alsoub, H., \& Chacko, K. C. (1995). Descending necrotising mediastinitis. Postgraduate medical journal, 71(832), 98-101.

4. Petitpas, F., Blancal, J. P., Mateo, J., Farhat, I., Naija, W., Porcher, R., ... \& Mebazaa, A. (2012). Factors associated with the mediastinal spread of cervical necrotizing fasciitis. The Annals of thoracic surgery, 93(1), 234-238.

5. Chinh, N. D., Van Trung, P., Bang, P. H., Chuong, P. L., \& Tuy, N. M. (2003, October). Prise en charge des médiastinites et des abcès médiastinaux: à propos de 56 observations. In Annales de chirurgie (Vol. 128, No. 8, pp. 570-571). Elsevier Masson.

6. Razafimanjato, N. N. M., Raoelijaona, L., \& Rasoamampianina, L. E. (2012). Médiastinite compliquant une cellulite cervicale d'origine dentaire, à propos d'un cas et revue de la littérature. Rev. anesth réanim med Urgence, 4(1), 20-22.

7. La Rosa J, Bouvier S, Langerona O. (2008). Prise en charge des cellulites maxillofaciale. Prat Anesth Réa: 309-315.

8. Foroulis, C. N., \& Sileli, M. N. (2011). Descending necrotizing mediastinitis: review of the literature and controversies in management. Open Surg J, 5, 12-18.

9. Serghini I, Aissaoui Y, Quamouss Y, Sedikki R, Filali K, Zoubir M, et al. (1995). Mediastinitis complicating a cervical cellulitis of dental gateway: report of a case and literature review. Clin Infect Dis : 51-6.

10. Mathieu, D., Neviere, R., Teillon, C., Chagnon, J. L., Lebleu, N., \& Wattel, F. (1995). Cervical necrotizing fasciitis: clinical manifestations and management. Clinical infectious diseases, 21(1), 51-56.

11. Giudicelli, R., Doddoli, C., Velly, J. F., Brichon, P. Y., Jancovici, R., \& Pons, F. (2003). Les médiastinites nécrosantes descendantes: à propos de 30 observations. J Chir Thorac Cardiovasc, 7, 69-72.

12. Hirai, S., Hamanaka, Y., Mitsui, N., Isaka, M., \& Mizukami, T. (2004). Surgical treatment of virulent descending necrotizing mediastinitis. Annals of thoracic and cardiovascular surgery, 10(1), 34-38. 
13. Durrleman, N., El Hamamsy, I., Hébert, Y., Pellerin, M., Carrier, M., \& Perrault, L. P. (2006). Modalités thérapeutiques des médiastinites en chirurgie cardiaque. Chir Thorac Cardiovasc, 10, 4-17. 